\title{
Review
}

\section{Pathogenesis of Secretory Otitis Media}

\author{
Chao Wen, Xiaoyu Wang, Taisheng Chen, Hongying Ruan, Peng Lin \\ Department of Otorhinolaryngology Head and Neck Surgery, Tianjin First Center Hospital, TianJin, China
}

\author{
Keywords \\ Secretory otitis media; Infection; Immune; \\ Pathogenesis
}

Correspondence

Peng Lin, E-mail: wwcc_0512@163.com

DOI: 10.1515/ii-2017-0098

\begin{abstract}
Secretory otitis media (SOM) is a common and frequently occurring disease featured by middle ear cavity exudant, ear nausea, and hearing loss. Morbidity of children is higher than that of adults. The pathogenesis and etiology of SOM are clear so far. Previous reports concluded that the mechanical obstruction and dysfunction of the eustachian tube are among the important causes of infection. The mechanism of infection and immune response in the pathogenesis of SOM is currently becoming a research hot spot, providing a reference for further study.
\end{abstract}

\section{Mechanical obstruction and dysfunction of eustachian tubes}

Cunsolo et al. ${ }^{[1]}$ reported that the mechanical obstruction and dysfunction of the eustachian tube prevent air from entering the middle ear. The middle ear cavity is in a negative pressure state, leading to middle ear mucosal hypoxia, telangiectasia, high permeability, and middle ear exudates. Ryding et al. ${ }^{[2]}$ observed 34 cases of 16- to 25-year-old chronic secretory otitis media (SOM) patients and also believed that the dysfunction of the eustachian tube is one of the pathogeneses of SOM. The surface active substance of the eustachian tube is one of the important pathogeneses for the dysfunction of the eustachian tube ${ }^{[3]}$. Yuan et al. ${ }^{[4]}$ observed the changes of morphology of the pharyngeal ostium of the eustachian tube and morphology around the pharyngeal ostium of the eustachian tube in 72 cases (144 ears) of the control group and 89 cases (151 ears) of the SOM group. Their study found that the morphological changes of the pharyngeal ostium of the eustachian tube among adults were associated with the occurrence and development of SOM. They also found that in the SOM group, all the children suffering from pathological changes around the pharyngeal ostium of the eustachian tube were affected by nasopharyngeal inflammation, followed by adenoid hypertrophy and eustachian tube tonsil hypertrophy, indicating that the morphological change of the pharyngeal ostium of the eustachian tube and its surrounding structural changes are associated with the occurrence of SOM.
Kanai et al. ${ }^{[5]}$ conducted a prospective study of 97 patients (180 ears) with nitrous oxide inhalation anesthesia and found that many patients were able to detect negative pressures in their middle ears and with general anesthesia, and 3.3\% of patients developed SOM. Therefore, they believed that the gas dynamics changes in the middle ear cavity caused by nitrous oxide inhalation anesthesia and transient eustachian tube obstruction were the major factors that led to general anesthesia postoperative SOM.

In recent years, some scholars ${ }^{[6]}$ proposed the concept of nasal SOM. In general, SOM is caused by nasal diseases (inflammation, tumor, allergies, and so on). Specifically, SOM is caused by the abnormality of the nasal structure. Lin Jie et al. ${ }^{[6]}$ diagnosed and treated 176 patients of SOM caused by abnormal nasal cavity and proposed the concept of nasal secretory otitis media. They advocated early conservative treatment. Patients with repeated relapse should be treated by surgical operation to correct the nasal cavity structure and restore nasal ventilation. The underlying reason may probably be an abnormal nasal structure that directly leads to the pharyngeal obstruction of the eustachian tube, blocked secretion discharge, sphenopalatine nerve, and nasopalatine nerve that can stimulate the mucous membrane distributed in the posterior part of the nasal cavity, paries lateralis of the nasal cavity, and posterior part of the nasal septum, which cause nasal congestion and dysfunction of ventilation and drainage of the eustachian tube. Abnormal nasal structure causes the obstruction of discharge of microorganisms and their secreted toxins. These microorganisms can retrograde 
to the middle ear cavity through the eustachian tube, leading to the occurrence of otitis media.

Obstructive sleep apnea hypopnea syndrome (OSAHS) is one of the most common respiratory obstructive diseases in children. Its hypertrophic adenoids can lead to eustachian tube dysfunction and to SOM through many ways, including the oppression of hypertrophic adenoid and blockage of the eustachian tube pharynx that results in the negative pressure of tympanic cavity following mucosal exudant. Moreover, the surface ciliated columnar epithelium of hypertrophic adenoid is converted into squamous epithelium and connective tissue fibrosis, which block the drainage of the discharge system of the eustachian tube and middle ear mucosa ${ }^{[7]}$. Adenoid is taken as a storage pool of pathogenic microbes. Hypertrophic adenoid can block the postnaris and lead to high nasopharyngeal pressure, allowing the secretion of the eustachian tube reflux into the middle ear. The abnormal immune function of adenoid can secrete histamine, which also causes mucosal edema of the eustachian tube ${ }^{[8]}$.

\section{Infection factors}

Although the mechanical obstruction and dysfunction of the eustachian tube have been identified as the important factors in the occurrence of SOM, this clinical work found that many patients with congenital small ear deformity associated with stenosis of the eustachian tube had no middle ear exudant. When bacteria or toxins were injected into the ear cavities of animals without obstruction and dysfunction of the eustachian tube, middle ear exudant occurred ${ }^{[9]}$. This finding showed that the mechanical obstruction and dysfunction of the eustachian tube are not the necessary conditions to develop SOM. The exudant in the middle ear cavity and the dysfunction of the eustachian tube cause middle ear exudant, which can develop into SOM. Literatures have suggested that a variety of bacteria, viruses, and Chlamydia has been separated from SOM middle ear exudant and thus are considered the factors for the occurrence and infection of SOM.

The common pathogenic bacteria associated with SOM are S. pneumoniae, H. influenza, and M. catarrhalis, followed by beta-hemolytic Streptococcus, Staphylococcus aureus, and so on. Furthermore, a variety of respiratory viruses can be isolated from the middle ear exudant of SOM patients, and the DNA of a variety of virus can be detected through PCR technology, suggesting that viruses often coexist with bacteria to cause diseases ${ }^{[10]}$. Brockson et al. ${ }^{[1]]}$ found that among the patients with acute bacterial otitis media, respiratory syncytial virus is the most common virus; thus, it is considered the most important common pathogenic pathogens. The role of $M$. catarrhalis in the pathogenesis of SOM has recently been elucidated, but further study has been hampered by the lack of animal models. The synergistic infection of respiratory syncytial virus and H.influenzae can help M. catarrhalis enter into the middle ear cavity, causing SOM.

Daniel et al. ${ }^{[12]}$ analyzed the middle ear exudant samples using the bacterial active staining technique and confocal laser scanning microscopy (CLSM) and found that the biofilm contained an average of 1.7 kinds of bacterial strains and two kinds of planktons; of which the most common bacterium was the coagulase negative Staphylococcus.

\section{Immune response}

In recent years, according to the clues of epidemiology, a large number of studies has been made on the correlation of allergic rhinitis, sinusitis, nasal polyps, asthma, and so on, and the theory of the consistency of upper and lower respiratory tract inflammation was put forward ${ }^{[13,14]}$. Animal experiments also confirmed that the proportion of immune cells in the middle ear mucosal system was similar to that in the nasal mucosa ${ }^{[15]}$. Therefore, as a continuation of upper respiratory tract mucosa, the middle ear mucosal system has the immune function that is similar to the nasal mucosa. Given the pathological factors, varieties of immune cells can proliferate and migrate, affecting the immune response.

Previous epidemiological study revealed that allergic reaction is one of the risk factors for the development of SOM. ${ }^{[16]}$ Among the allergic patients with SOM, mast cells, eosinophils, and $\mathrm{T}$ cells increased in their middle ear mucosa followed by excessive activation. The contents of immunoglobulin $\mathrm{E}(\mathrm{IgE})$, eosinophil cationic protein, and other allergic inflammatory mediators in the middle ear exudant increased and the expression of Thelper cells (Th)-2 type cytokines also significantly increased. Thus, Type I allergic reaction dominated by Th2 response may be one of the pathogenic factors, leading to persistent incurability of middle ear exudant among the allergic patients with $\mathrm{SOM}^{[17]}$. Nguyen et al.$^{[18]}$ also found that among the allergic patients with SOM, Th2-type immune response was characterized by increased eosinophils, $\mathrm{T}$ cells, and interleukin (IL) -4 in middle ear exudant, the eustachian tube mucosa, and nasopharyngeal mucosa. They also believed that allergic inflammations similar to allergic rhinitis and asthma were the pathogenic factors of allergic inflammation.

With the in-depth study of immune cells and their 
cytokine networks, scholars have proposed the Th1/Th2 equilibrium hypothesis: the body's response to antigen stimulation is achieved by the immune network system formed by the interaction of immune cells and their metabolites; $\mathrm{CD}^{+} \mathrm{T}$ cells can undergo polarization reactions with different antigens, generating two kinds of effector $\mathrm{T}$ cells (Th1 and Th2 cells) and can induce different immune responses. Moreover, Th1 cells interact with Th2 cells and regulate each other. On one hand, for the autoimmune and chronic infectious diseases, Th1 cells are involved in immunopathological effects through their specific transcription factors T-beta ${ }^{[19]}$ and autocrine cytokine [IL-2, interferon- $\gamma$, IFN- $\gamma$ ]. On the other hand, the main mediate allergic inflammations of Th2 cells ${ }^{[20]}$ can be controlled by their specific transcription factor GATA- ${ }^{[18]}$ and autocrine cytokine (IL-4, IL-5, IL-9, IL -13, and so on).

Zhao et al. ${ }^{[21]}$ studied the allergen-induced rat SOM model and IL-4. The IFN- $\gamma$ in the middle ear cavity lavaged the fluid of non-SOM group and Kappa B (nuclear transcription factors kappa $\mathrm{B}, \mathrm{NF}-\kappa \mathrm{B}$ ) in the middle ear mucosa and bone marrow cavity and found that the IL-4 synthesis in the middle microenvironment of SOM rats significantly increased; IFN- $\gamma$ decreased and Th2/Th1 ratio increased. Th polarization was characterized by the excessive differentiation of Th2 cells. NF- $\kappa B$ played a role in the regulation of Th1/Th2 polarization in the middle ear microenvironment of rats.

Kariya et al. ${ }^{[22]}$ analyzed the middle ear exudants of 80 cases of adult patients with otitis media. The detection rates of IL-2, IL-4, IL-5, IL-10, IL-12, and IFN- $\gamma$ were 75\% (60 cases), $41.3 \%$ (33 cases), $52.5 \%$ (42 cases), $17.5 \%$ (14 cases), $100 \%$ ( 80 cases), and $82.5 \%$ (66 cases), respectively. They believed that whether or not they are prone to be allergic, IL-12 plays an important role in the pathogenesis of SOM by affecting the generation of IL- 2 and IFN- $\gamma$. IL- 4 had an effect on the immune status of patients with allergic rhinitis. IL-10 may have affected the viscosity of middle ear exudant.

Smirnova et al. ${ }^{[17]}$ found that the interaction of IL-2 and IL-4 played an important role in the chronic phase of inflammatory response. The Th2-related cytokines IL-4, IL-5, and IL-13 and the Th2/Th1-related cytokine granulocyte and granulocyte-macrophage colonystimulating factor (GM-CSF) were involved in the cytological and molecular regulation of the chronic inflammation of middle ear and subsequent SOM. Skotnicka et al. ${ }^{[23]}$ detected the peripheral blood and $\mathrm{T}$ lymphocyte subsets in the middle ear exudants of patients with chronic SOM through flow cytometry and found that $\mathrm{CD}^{+} \mathrm{T}$ cells increased in middle ear exudant. Compared with peripheral blood $\mathrm{T}$ cell subsets, the numbers of $\mathrm{CD}^{+}, \mathrm{CD}^{+} \mathrm{T}$ cells, and $\mathrm{CD} 4^{+} / \mathrm{CD}^{+}$ratio increased significantly. Thus, they speculated that some factors in the middle ear can regulate the proliferation and differentiation of lymphocytes. Cooter et al. ${ }^{[24]}$ found that the middle ear exudant of all patients with chronic SOM contained transforming growth factor- $\beta$, TGF- $\beta$ (TGF- $\beta 1$ and TGF- $\beta 2$ ), and the expressions of TGF- $\beta 1$ and TGF- $\beta 2$ in the middle ear exudant of patients with chronic SOM and tympanostomy tube insertion increased. They also speculated that TGF- $\beta$ is closely related to the chronic progression of SOM and may play a key role in the formation of the fibrosis of SOM complications, including gel ear and adhesive otitis media. However, there is still lack of conclusive evidence. Li Jie et al. made an analysis on middle ear exudant and the peripheral blood of chronic SOM patients and found that TGF- $\beta 1$ plays an important role in the chronic progression of SOM.

Previous studies showed that regulatory Tlymphocyte (Treg) plays a role in the human immune response ${ }^{[26]} \cdot \mathrm{CD}^{+}$ $\mathrm{CD} 25^{+}$Treg, as a functional mature $\mathrm{T}$ cell subtype, can play an immunosuppressive function through direct contact between cells and secretion of inhibitory cytokines TGF- $\beta$ and IL-10 ${ }^{[27]}$. As a housekeeping gene of $\mathrm{CD} 4{ }^{+} \mathrm{CD} 25^{+}$Treg, Foxp3 is the specific maker of such cellular activation ${ }^{[28]}$. In vitro experiments suggested that Foxp $3^{+}$Tregs had immunosuppressive functions. T cells had no reactivity and inhibition, and genetic defects led to immune tolerance defect and immune dysfunction ${ }^{[26]}$. Recent studies have shown that cytokine TGF- $\beta$ can be used as a physiological regulation factor of Foxp3 induction, which can prevent $\mathrm{T}$ cell activation through an inhibitory mechanism ${ }^{[29]}$.

\section{Others}

\section{Aquaporins}

To maintain the air filling and liquid efflux in the middle ear cavity, the eustachian tube is needed to have the function of better ventilation and excretion, whereas all the membrane structures in the tympanic cavity should have the function of water molecule absorption. This kind of absorption function mainly relies on the eustachian tube, aquaporin (AQP), and ion channels on the cell membrane of tympanic epithelium, forming a permeation gradient through the direct transport of $\mathrm{Na}^{+}, \mathrm{K}^{+}$and the transport of water molecules and other ions, thereby allowing the liquid to absorb and maintain the steady state of water to prevent the occurrence of middle ear exudant ${ }^{[27]}$. AQP is considered an important structure to 
maintain the balance of water in the middle ear ${ }^{[30]}$. When the pathogen invades and destroys the steady state in the middle ear, the ion channel is affected, and the flow state of ions is changed, resulting in a change in the function and expression of transmembrane AQPs. The abnormalities of AQPs can cause abnormalities in intracellular signals and changes in cell membrane potential, resulting in exudation ${ }^{[31]}$.

Zhang et al. ${ }^{[32]}$ detected the animal model of SOM group and AQP4 and AQP5 in the tympanic mucosa of the normal control group through RT-PCR and Western blot experiment. Quantitative analysis showed that the expression of AQP4 and AQP5 in SOM group was higher than that in the control group; hence, they believed that AQP4 and AQP5 in the middle ear cavity of SOM patients can play an important role in maintaining homeostasis and preventing from middle ear exudant.

\section{Hypoxia inducible factor}

The signaling pathways of hypoxia-inducible factor-vascular endothelial growth factor (HIF-VEGF), tympanic membrane puncture, and tympanic membrane catheterization are commonly used to treat SOM. As hypoxia is a common feature of inflammatory response, this treatment is featured by increasing middle ear ventilation to relieve hypoxia in the middle ear, correcting negative pressure and preventing fluid formation ${ }^{[3,34]}$. Hypoxia response can be regulated by hypoxia inducible factor (HIF) protein. HIF is a transcription factor. Its induced product can restore blood supply, promote nutrition, and generate energy, thus maintaining the steady state of tissues. In the case of normal oxygen content, HIF-1a can be modified and degraded by prolyl hydroxylase domain (PHD). In the case of hypoxia, PHD activity is limited; HIF-1a structure is stable. The latter forms a heterodimer with HIF-1b before entering the nucleus and combining with hypoxia response element ${ }^{[35]}$. Moreover, HIF signal can be regulated through the interaction of HIF$\mathrm{la}$ and $\mathrm{NF}-\mathrm{Kb}$ at the transcription level. At the translation level, HIF signal can be regulated through cytokines, including IL-1b and TNF- $\alpha^{[33,34]}$. HIF response can adapt to and improve ischemic status ${ }^{[36,37]}$ and can regulate the immune response caused by microbial infection. In the case of chronic hypoxic inflammatory response, vascular endothelial growth factor (VEGF) induced by HIF can promote the formation of neovascularization ${ }^{[38]}$, leading to the disorder of signal regulation and pathological change ${ }^{[39]}$ and the occurrence of related diseases. Animal experiments have confirmed that the expression of HIF-1a in the middle ear mucosa of SOM rats increased ${ }^{[40]}$. Given that the HIF-
VEGF signaling pathway plays an important role in the process of SOM formation, the study on relevant factors provides the possibility of SOM treatment ${ }^{[41]}$.

\section{Obesity}

To evaluate the relationship between SOM children patients and body mass index (BMI), Kim et al..$^{[42]}$ examined 140 patients with SOM from 2 to 7 years of age with surgery of unilateral or bilateral tympanic membrane and 190 nonSOM patients without routine surgery of the ear. Result showed that the prevalence of obesity in the experimental group was higher than that in the control group $(P<0.05)$. However, the BMI, triglyceride, and total cholesterol between the two groups had no significant difference. Kim et al. also thought that childhood obesity has a certain effect on the development of SOM, but there is no correlation with the occurrence of SOM.

The pathogenesis of SOM is complicated and diverse. The roles of mechanical obstruction and dysfunction of the eustachian tube, infection, and immune response in the pathogenesis of SOM have been confirmed. In recent years, studies on the etiology of molecules and genes have made rapid progress. The entire pathogenic network has been expanded; hence, further study on etiology can provide the basis for SOM treatment.

\section{Declarations}

\section{Acknowledgements}

No.

\section{Competing interests}

The authors declare that they have no competing interest.

\section{Authors' contributions}

C Wen, XY Wang and TS Chen made the literature analysis and wrote, discussed and revised the manuscript of this review. HY Ruan and P Lin critically analyzed and corrected the manuscript. All authors read and approved the final manuscript.

\section{References}

1 Cunsolo E, Marchioni D, Leo G, et al. Functional anatomy of the eustachian tube. Int J Immunopathol Pharmacol, 2010, 23(1 Suppl): 4-7.

2 Ryding $\mathrm{M}$, White $\mathrm{P}, \mathrm{Kalm} \mathrm{O}$. Eustachian tube function and tympanic membrane findings after chronic secretory otitis media. Int J Pediatr Otorhinolaryngol, 2004, 68(2): 197-204. 
3 Ma Z, Chen X, Li M, et al. Progress in the treatment of secretory otitis media with surfactant. Ting Li Xue Ji Ji Bing Yan Wu Ji Bing Za Zhi, 2003, 11: 67 .

4 Yuan Y, LI G, Zhou W, et al. Study on the relationship between ostium pharyngeum tubae auditivae and its surrounding morphological changes and secretory otitis media. Lin Chuang Er Bi Hou Tou Jing Wai Ke Za Zhi, 2007, 21: 1024.

$5 \quad$ Kanai R, Kaneko K. Negative middle ear pressure and otitis media with effusion after surgery under general anesthesia. Acta Otolaryngol, 2012, 132(10): 1049-1053.

6 Lin J, Mu Z, Yu G, et al. Diagnosis and treatment of nasal secretory otitis media. Lin Chuang Er Bi Hou Tou Jing Wai Ke Za Zhi, 2011, 25: 301.

7 Yasan $\mathrm{H}$, Dogru $\mathrm{H}$, Tuz $\mathrm{M}$, et al. Otitis media with effusion and histopathologic properties of adenoid tissue. Int J Pediatr Otorhinolaryngol, 2003, 67(11): 1179-1183.

8 Becker S, Koch T, Philipp A. Allergic origin of recurrent middle ear exudant and adenoids in young children. HNO, 1991, 39(5): 182-184.

9 Takahashi H, Fujita A, Lee SH, et al. Experimental conditions for the development of persistent otitis media with effusion. Eur Arch Otorhinolaryngol,1990, 247(2): 89-92.

10 Zhang Z, Liu H. Pathogenesis of secretory otitis media. Yi Xue Zong Shu, 2004, 10: 751 .

11 Brockson ME, Novotny LA, Jurcisek JA, et al. Respiratory syncytial virus promotes moraxella catarrhalis-induced ascending experimental otitis media. PLoS One, 2012, 7(6): e40088.

12 Daniel M, Imtiaz-Umer S, Fergie N, et al. Bacterial involvement in otitis media with effusion. Int J Pediatr Otorhinolaryngol, 2012, 76(10): 1416-1422.

13 Grossman J. One airway, one disease. Chest, 1997, 111(2 Suppl): 11S-16S.

14 Gu Z. Respiratory tract inflammatory response. Zhonghua Er Bi Yan Hou Ke Za Zhi, 2001, 36: 80.

15 Suenaga S, Kodama S, Ueyama S, et al. Mucosal immunity of the middle ear: analysis at the single cell level. Laryngoscope, 2001, 111(2): 290-296.

16 Alles R, Parikh A, Hawk L, et al. The prevalence of atopic disorders in children with chronic otitis media with effusion. Pediatr Allergy Immunol, 2001, 12(2): 102-106.

17 Smirnova MG, Birchall JP, Pearson JP. Evidence of T-helper cell 2 cytokine regulation of chronic otitis media with effusion. Acta Otolaryngol, 2005, 125(10): 1043-1050.

18 Nguyen LH, Manoukian JJ, Sobol SE, et al. Similar allergic inflammation in the middle ear and the upper airway: evidence linking otitis media with effusion to the united airways concept. J Allergy Clin Immunol, 2004, 114(5): 1110-1115.

19 Ishizaki K,Yamada A,Yoh K, et al. Th1 and type 1 cytotoxic T cells dominate responses in T-bet overexpression transgenic mice that develop contact dermatitis. J Immunol, 2007, 178(1): 605-612.

20 Leigh R, Ellis R, Wattie JN, et al. Type 2 cytokines in the pathogenesis of sustained airway dysfunction and airway remodeling in mice. Am J Respir Crit Care Med, 2004, 169(7): 860-867.
21 Zhao S, Liu H, Han D, et al. Expression of middle ear nuclear factor $\kappa B$ in exudative otitis media of rats induced by allergen. Lin Chuang Er Bi Hou Tou Jing Wai Ke Za Zhi, 2008, 22: 751.

22 Kariya S, Okano M, Hattori $\mathrm{H}$, et al. TH1/TH2 and regulatory cytokines in adults with otitis media with effusion. Otol Neurotol, 2006, 27(8): 1089-1093.

23 Skotnicka B, Stasial-Barmuta A, Hassmann-Poznanske E, et al. Lymphocyte subpopulations in middle ear exudant: flow cytometry analysis. Otol Neurotol, 2005, 26(4): 567-571.

24 Cooter MS, Eisma RJ, Burleson JA, et al. Transforming growth factorbeta expression in otitis media with effusion. Laryngoscope, 1998, 108(7): 1066-1070.

$25 \mathrm{Li} \mathrm{J}$, Zhao S, Liu H, et al. Clinical significance of detection of TGF- $\beta 1$ in middle ear exudant of secretory otitis media. Ting Li Xue Ji Ji Bing Yan Wu Ji Bing Za Zhi, 2008, 16: 383.

26 Daniele N, Scerpa MC, Landi F, et al. T (reg) cells: collection, processing, storage, and clinical use. Pathol Res Pract, 2011, 207(4): 209-215.

27 Joetham A, Takada K, Taube C, et al. Naturally occurring lung CD4+, $\mathrm{CD}_{25} 5^{+} \mathrm{T}$ cell regulation of airway allergic responses depends on IL-10 induction of TGF-beta. J Immunol, 2007, 178(3): 1433-1442.

28 Mall M, Grubb BR, Harkema JR, et al. Increased airway epithelial Na absorption produces cystic fibrosis-like lung disease in mice. Nat Med, 2004, 10(5): 487-493.

29 Verkman AS, Mitra AK. Structure and function of aquaporin water channels. Am J Physiol Renal Fluid Electrolyte Physiol, 2000, 278: F13.

30 Lim DJ, Chun YM, Lee SK, et al. Cell biology of tubotympanum in relation to pathogenesis of otitis media-a review. Vaccine, 2001, $19 \mathrm{Suppl}$ 1: S17-S25.

31 Zhang Q, Liu C, Wang J, et al. Expression pattern of aquaporin 4 and 5 in the middle ear of guinea pigs with secretory otitis media. Acta OtoLaryngologica, 2010, 130(1): 68-74.

32 Frede S, Berchner-Pfannschmidt U, Fandrey J. Regulation of hypoxiainducible factors during inflammation. Meth Enzymol, 2007, 435: 405-419.

33 Dehne N, Brune B. HIF-1 in the inflammatory microenvironment. Exp Cell Res, 2009, 315(11): 1791-1797.

34 Doedens A, Johnson RS. Transgenic models to understand hypoxiainducible factor function. Meth Enzymol, 2007, 435: 87-105.

35 Rius J, Guma M, Schachtrup C, et al. NF-kB links innate immunity to the hypoxic response through transcriptional regulation of HIF-1a. Nature, 2008, 453(7196): 807-811.

36 Taylor CT. Interdependent roles for hypoxia inducible factor and nuclear factor-kappaB in hypoxic inflammation. J Physiol, 2008, 586(17): 4055-4059.

37 Oliver KM, Taylor CT, Cummins EP. Hypoxia regulation of NFkappaB signaling during inflammation: the role of hydroxylases. Arthritis Res Ther, 2009, 11(1): 215 .

38 Zinkernagel A, Johnson R, Nizet V. Hypoxia inducible factor (HIF) function in innate immunity and infection. J Mol Med (Berl), 2007, 
85(12): 1339-1346.

39 Huang Q Cai X, Zhang Z, et al. Expression of hypoxia-inducible factor in otic mucosa of rats with secretory otitis media. Ting Li Xue Ji Ji Bing Yan Wu Ji Bing Za Zhi, 2010, 18: 63.

40 Cheeseman MT, Tyrer HE, Williams D, et al. HIF-VEGF pathways are critical for chronic otitis media in junbo and jeff mouse mutants. PLoS Genet, 2011, 7: e1002336.

41 Kim SH, Park DC, Byun JY, et al. The relationship between overweight and otitis media with effusion in children. Int J Obes (Lond), 2011, 35(2): 279-282. 